\title{
EXOSPHERIC TEMPERATURE OF VENUS FROM \\ MARINER 5
}

\author{
CHARLES A. BARTH \\ Dept. of Astro-Geophysics and Laboratory for Atmospheric and Space Physics, University of \\ Colorado, Boulder, Colo., U.S.A.
}

\begin{abstract}
The Lyman alpha measurements made on the sunlit side of Venus by Mariner 5 showed that the distribution of the radiating hydrogen atoms was governed by two scale heights. A revised model is presented in which the inner or cold distribution is attributed to hydrogen atoms in thermal equilibrium with the thermosphere and the outer or hot distribution is due to hydrogen atoms produced by the photodissociation of molecular hydrogen. The consequence of this interpretation is that the temperature of the dayside thermosphere of Venus is $325 \mathrm{~K}$.
\end{abstract}

\section{Introduction}

The ultraviolet photometer data from Mariner 5 shows that the Lyman alpha emitters in the upper atmosphere of Venus must be present either in two forms or at two different temperatures (Barth, 1968). Among the several models suggested, one requires the presence of both molecular and atomic hydrogen (Barth et al., 1968) while the other has hydrogen atoms present in both a thermal and a 'hot' population. At the time of the original suggestion (Barth, 1968), there was no plausible physical model for producing the 'hot' atoms. The present paper offers a model in which the molecular hydrogen model and the 'hot' atom model are combined. In this theory, the 'hot' atoms arise from the photodissociation of molecular hydrogen.

Since there is water vapor in the atmosphere of Venus, there should be molecular hydrogen as well, just as there is molecular hydrogen in the earth's upper atmosphere. Lewis $(1968,1970)$ has calculated that under chemical equilibrium conditions in the dense, lower atmosphere of Venus, the reaction $\mathrm{CO}+\mathrm{H}_{2} \mathrm{O}=\mathrm{CO}_{2}+\mathrm{H}_{2}$ leads to a mixing ratio for molecular hydrogen of the order of $10^{-6}$. In the upper atmosphere, diffusive separation should set in and molecular hydrogen may become the major atmospheric constituent over a limited altitude range. In the region where the molecular hydrogen is no longer screened from the solar ultraviolet radiation by carbon dioxide, photodissociation will occur.

\section{Photodissociation of Molecular Hydrogen}

Molecular hydrogen is photodissociated by ultraviolet radiation shortward of $845 \AA$ into two hydrogen atoms.

$$
\mathrm{H}_{2}+h v(\lambda<845 \AA) \rightarrow \mathrm{H}(1 s)+\mathrm{H}(2 s, 2 p)
$$

This direct photodissociation produces half of the atoms in the $1 s$ state and the other half in either the $2 s$ or $2 p$ depending upon the particular photodissociation continua 
followed. Dalgarno and Allison (1969) have calculated the cross sections for photodissociations proceeding by way of the $B^{1} \Sigma_{u}^{+}, C^{1} \pi_{u}$, and $B^{1} \Sigma_{u}^{+}$states.

Photodissociations that are caused by photons with energy greater than $14.7 \mathrm{eV}$ (the photodissociation threshold) will produce atoms with excess kinetic energy. In this way, the photodissociation of molecular hydrogen in the exosphere of Venus, where collisions do not occur, will produce a population of hot atoms.

The rate of production of hydrogen atoms from the photodissociation of molecular hydrogen is given by the following expression, since in the exosphere, densities are sufficiently low that there is no attenuation of the solar radiation:

$$
[\dot{\mathrm{H}}]=2\left[\mathrm{H}_{2}\right] \sum_{\lambda<845 \AA} \sigma_{\lambda}\left(\mathrm{H}_{2}\right) F_{\lambda}
$$

where the square brackets designate volume densities of the chemical constituent within the bracket, $\sigma_{\lambda}\left(\mathrm{H}_{2}\right)$ is the photodissociation cross-section of molecular hydrogen, and $F_{\lambda}$ is the solar flux. Since the last two quantities are functions of wavelength, their product needs to be summed for wavelengths less than $845 \AA$, the photodissociation threshold. The total flux of photodissociated hydrogen atoms may be obtained by integrating the rate of photodissociation from the base of the exosphere to infinity. Using the continuity equation, this flux of newly formed atoms may be equated to a volume density of hydrogen atoms times an average velocity.

$$
\int_{n}^{\infty}[\dot{\mathrm{H}}] \mathrm{d} z=[\mathrm{H}] \bar{\nu}
$$

This average velocity represents the excess kinetic energy shared by the atoms in the photodissociation process, and is a function of the wavelength of the photon. The flux of photodissociated atoms may be equated to the photodissociation process by integrating the molecular hydrogen density over altitude. This integral may be approximated by the local molecular hydrogen density times the molecular hydrogen scale height, $\mathscr{H}\left(\mathrm{H}_{2}\right)$.

The density of freshly photodissociated hydrogen atoms at the base of the exosphere may be related to the density of molecular hydrogen at the same level through the following equation

$$
[\mathrm{H}]=2 \sum\left(\frac{\sigma_{\lambda}\left(\mathrm{H}_{2}\right) F_{\lambda}}{\bar{\nu}}\right) \mathscr{H}\left(\mathrm{H}_{2}\right)\left[\mathrm{H}_{2}\right]
$$

The quantities in the large parentheses including the average velocity are all functions of the wavelength of the photodissociating photon and should be calculated separately and then summed. Using the solar fluxes of Hinteregger et al. (1965), adjusted by a factor of 1.5 to make them appropriate to October 1967, and the photodissociation cross sections of Dalgarno and Allison (1969), the atom production rates and the average excess energy of the freshly dissociated atoms are given in Table I. In Equation (4), the density of atomic hydrogen that has been converted from molecular hydrogen is given in terms of the molecular hydrogen density. This 'conversion factor' has been 
TABLE I

\begin{tabular}{lllll}
\hline Wavelength & $\begin{array}{l}\text { Average } \\
\text { excess energy }\end{array}$ & $\begin{array}{l}\text { Solar flux } \\
\text { Venus, } 1967\end{array}$ & $\begin{array}{l}\text { Production } \\
\text { rate }\end{array}$ & $\begin{array}{l}\text { Conversion } \\
\text { factor }\end{array}$ \\
\hline$\AA$ & $\mathrm{eV}$ & $10^{9} \mathrm{~cm}^{-2} \mathrm{sec}^{-1}$ & $10^{-9} \mathrm{p} \mathrm{sec}^{-1}$ & $10^{-7}$ \\
\hline $840-830$ & 0.08 & 2.1 & 19.3 & 7.93 \\
$832-835$ & 0.10 & 1.5 & 13.8 & 5.01 \\
$830-820$ & 0.17 & 1.9 & 14.4 & 4.05 \\
$820-810$ & 0.27 & 1.7 & 9.9 & 2.19 \\
$810-796$ & 0.38 & 2.0 & 9.2 & 1.71 \\
790.1 & 0.51 & 1.0 & 2.8 & 0.45 \\
787.7 & 0.53 & 0.92 & 2.6 & 0.40 \\
780.3 & 0.60 & 0.44 & 1.0 & 0.14 \\
$796-780$ & 0.53 & 2.1 & 5.9 & 0.93 \\
770.4 & 0.71 & 1.2 & 2.0 & 0.27 \\
765.1 & 0.76 & 0.60 & 0.8 & 0.10 \\
$780-760$ & 0.71 & 2.1 & 3.6 & 0.50 \\
\hline & & & & \\
\hline
\end{tabular}

calculated as a function of average excess energy and is listed in Table I also. The conversion factor summed over all energies which gives the amount of atomic hydrogen at a given altitude that has been converted from molecular hydrogen is $2.4 \times 10^{-6}$. Each of the subgroups of hydrogen atoms will be distributed with their own scale height depending on their average kinetic energy. This calculated value for the conversion factor is a minimum since some processes that photodissociate molecular hydrogen have been neglected. Mentall and Gentieu (1969) have found that absorption into the $D^{1} \pi_{u}$ state leads to predissociation producing additional hydrogen atoms. A more important one is the photo-destruction mechanism proposed by Stecher and Williams (1967) in which ultraviolet radiation shortward of $1108 \AA$ is absorbed into discrete excited states and then reradiated into the continuum of the ground state producing dissociation. Dalgarno and Allison (1969) have estimated that this process may produce ten times as many atoms as the photodissociation process calculated above.

\section{The Model}

This model identifies the Lyman alpha emission from the outer atmosphere of Venus as arising from the resonance scattering of solar Lyman alpha radiation by atomic hydrogen. The emissions originating from planetocentric distances less than $9000 \mathrm{~km}$ come from thermal hydrogen atoms; i.e., atoms that have undergone collisions below the exobase and are in thermal equilibrium with the constituents of the thermosphere. The Lyman alpha emissions arising from planetocentric distances greater than $9000 \mathrm{~km}$ originate from 'hot' hydrogen atoms, i.e., atoms that have been produced by the photodissociation of molecular hydrogen in the exosphere and have not undergone any collisions since their formation. This identification of the observations with 'hot' 
and thermal hydrogen atoms is illustrated in Figure 1 which has been adopted from a figure used for an alternate model (Barth, 1968).

The identification of the lower altitude regime of the data with thermal hydrogen atoms leads to the requirement that the thermospheric temperature of Venus be $325 \mathrm{~K}$. The bottom of the exosphere, the exobase, will be located at the level above which collisions essentially do not take place. The cross section for thermalizing collisions between hydrogen atoms and hydrogen molecules may be estimated to be $3.5 \times 10^{-16}$ $\mathrm{cm}^{2}$. This means that the volume density of molecular hydrogen at the exobase can be no more than $2 \times 10^{8}$ molecules $\mathrm{cm}^{-3}$ since the molecular hydrogen scale height at $325 \mathrm{~K}$ is $160 \mathrm{~km}$. The carbon dioxide volume density at this same level needs to be $4 \times 10^{9}$ molecules $\mathrm{cm}^{-3}$ or less. The distribution of these additional constituents in the model are illustrated in Figure 2 where the curve marked atomic hydrogen contains both the thermal and 'hot' populations from Figure 1.

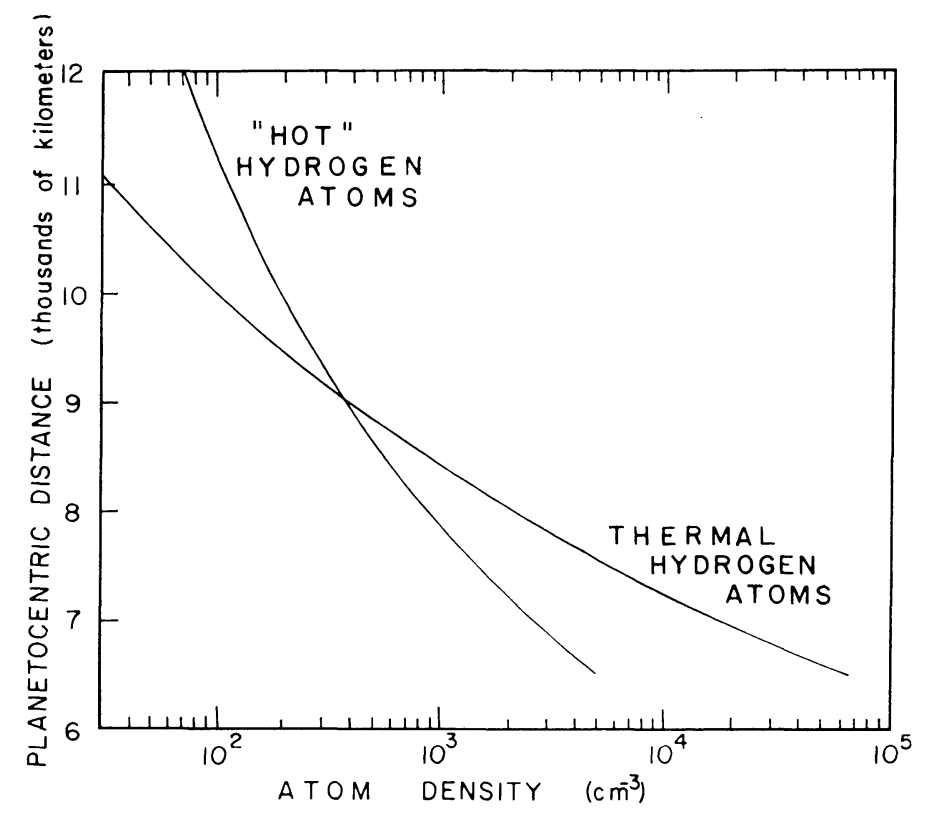

Fig. 1. Distribution of 'hot' and thermal hydrogen atoms in model.

\section{Test of Model}

When the conversion factor determined in the Table is applied to molecular hydrogen density at the exobase, the density of 'hot' hydrogen atoms at this level is calculated to be $5 \times 10^{2}$ atoms $\mathrm{cm}^{-3}$. The values of excess kinetic energy listed in the table indicate that the bulk of these atoms will have scale heights that exceed the $640 \mathrm{~km}$ scale height of the hot atom distributions in the model shown in Figure 1. This means that the 'hot' atom distribution at planetocentric distances less than $9000 \mathrm{~km}$ actually is less than that indicated in Figure 1 and at distances greater than $9000 \mathrm{~km}$ the distribution is greater than that shown in Figures 1 and 2. 


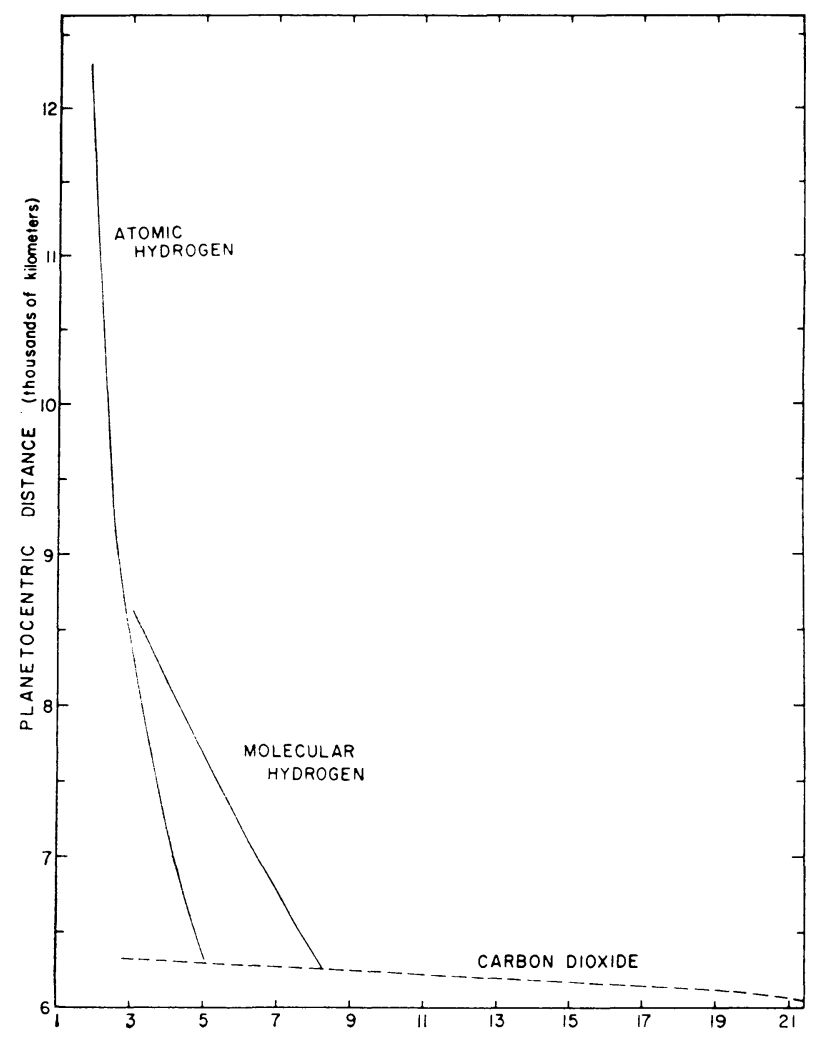

Fig. 2. Distribution of atomic and molecular hydrogen and carbon dioxide in model. Atomic hydrogen distribution includes both the 'hot' and thermal atoms shown in Figure 1.

When the Stecher-Williams process is taken into account, the conversion factor is ten times larger and the density of 'hot' atoms shown in Figure 1 can be matched using a scale height of $640 \mathrm{~km}$. To exactly match the distribution in the figure, the bulk of the 'hot' atoms would need to have an average energy that is twice the kinetic energy of the thermal atoms.

\section{Summary}

The molecular hydrogen model of Barth (1968) failed to take into account the resonance scattering of Lyman alpha radiation by the freshly created hydrogen atoms. When these 'hot' atoms are included, the Lyman alpha emissions at distances greater than $9000 \mathrm{~km}$ are accounted for. The Lyman alpha emissions at distances less than $9000 \mathrm{~km}$ are then attributed to thermal hydrogen atoms with the consequences that the Venus thermosphere is very cold, $325 \mathrm{~K}$. As compared with the earlier model, the amount of molecular hydrogen in this model is greatly reduced with the consequence that the Lyman alpha radiation that is radiated during the photodissociation process no longer plays a role in the explanation of the observations. 


\section{Acknowledgments}

The author appreciates the comments and suggestions made by Professor A. Dalgarno following the presentation of this paper at the symposium. This work has been supported by the National Aeronautics and Space Administration, NASA grant NGL-06-003-052.

\section{References}

Barth, C. A.: 1968, 'Interpretation of the Mariner 5 Lyman Alpha Measurements', J. Atmospheric Sci. 25, 564.

Barth, C. A., Wallace, L., and Pearce, J. B.: 1968, 'Mariner 5 Measurement of Lyman Alpha Radiation Near Venus', J. Geophys. Res. 73, 2541.

Dalgarno, A. and Allison, A. C.: 1969, 'Photodissociation of Molecular Hydrogen on Venus', $J$. Geophys. Res. 74, 4178.

Hinteregger, H. E., Hall, L. A., and Schmidtke, G.: 1965, 'Solar XUV Radiation and Neutral Particle Distribution in July 1963 Thermosphere', Space Res. 5, 1175.

Lewis, John S.: 1968, 'An Estimate of the Surface Conditions of Venus', Icarus 8, 434.

Lewis, John S.: 1970, 'Geochemistry of the Volatile Elements on Venus', Icarus 11, 367.

Mentall, J. E. and Gentieu, E. P.: 1969, 'Lyman- $\alpha$ Fluorescence From the Photodissociation of $\mathrm{H}_{2}$, GSFC X-616-69-497.

Stecher, Theodore, P. and Williams, David A.: 1969, 'Photo-destruction of Hydrogen Molecules in $\mathrm{H}_{1}$ Regions', Astrophys. J. 149, L29. 